\title{
Optimal gauge for the multimode Rabi model in circuit QED
}

\author{
Marco Roth, ${ }^{1,2}$ Fabian Hassler, ${ }^{2}$ and David P. DiVincenzo ${ }^{1,2}$ \\ ${ }^{1}$ JARA-Institute for Quantum Information (PGI-11), Forschungszentrum Jülich, 52428 Jülich, Germany \\ ${ }^{2}$ JARA-Institute for Quantum Information, RWTH Aachen University, 52056 Aachen, Germany
}

(Received 2 May 2019; published 25 November 2019)

\begin{abstract}
In circuit QED, a Rabi model can be derived by truncating the Hilbert space of an anharmonic qubit coupled to a resonator. This truncation breaks the gauge invariance present in the full Hamiltonian. Here we derive a simple criterion for an optimal gauge such that the differences between the truncated and the full Hamiltonian are minimized. We find that it is determined by the ratio of the anharmonicity of the qubit to an averaged resonator frequency. We demonstrate that the usual choices of flux and charge gauge are not necessarily the preferred options in the case of multiple resonator modes.
\end{abstract}

DOI: 10.1103/PhysRevResearch.1.033128

\section{INTRODUCTION}

Circuit QED [1,2] is a central subject of quantum information science that has deepened our understanding of light-matter interaction [3-5]. Most implementations feature a two-level system (qubit) that is coupled to a linear resonator. The qubit is formed by the two lowest energy levels of an anharmonic multilevel system. For the physics of interest, only the qubit subspace is important. The Schrieffer-Wolff (SW) transformation [6,7] is the standard method to perturbatively derive an effective Hamiltonian description within this subspace. For most purposes, it is sufficient to consider the effective Hamiltonian only to first order, yielding the well known quantum Rabi model (QRM). However, since the Hamiltonian of the nontruncated system is unique only up to a unitary transformation, the effective description is gauge dependent to every finite order [8,9]. This gauge ambiguity becomes particularly important in the (ultra)strong-coupling regime. It has been found that the QRM derived in a gauge where the qubit-resonator coupling is mediated by the flux variables leads to different predictions than the one where the coupling is mediated by the charge variables [10-12]. For example, it has been shown that the QRM derived in the charge gauge may completely fail to predict the energy spectrum of a fluxonium qubit [10].

In this work, we look at the issue from a different perspective. We use the gauge degree of freedom to find an optimal gauge such that the results of the effective model are as close as possible to the full model. Importantly, we take account of the need for a multimode description [13-17] in the quest to achieve the ultrastrong-coupling regime [18-21]. To increase the flexibility, we not only consider the extremal cases of purely flux or charge-mediated coupling, but perform a general gauge transformation that smoothly interpolates

Published by the American Physical Society under the terms of the Creative Commons Attribution 4.0 International license. Further distribution of this work must maintain attribution to the author(s) and the published article's title, journal citation, and DOI. between the two. A similar transformation has been used in [22] to extend the Jaynes-Cummings model into the ultrastrong-coupling regime.

We are interested in a situation where the system is described by a QRM, a two-level qubit coupled to many oscillator levels. In this case, a large positive anharmonicity is required to avoid coupling to higher qubit levels. We find that the second-order term of the effective Hamiltonian within the SW method is a good indicator of the validity of the QRM. Based on this observation, we derive a simple analytical criterion for the optimal gauge and benchmark it against numerical simulations of the full problem. For a strongly anharmonic qubit resonantly coupled to a single-mode resonator, the flux gauge is always the best gauge $[10,11,20]$. This serves as an analog of the dipole gauge in quantum optics [23]. Considering more than one mode drastically changes this simple picture. The optimal gauge may now deviate from the pure flux gauge as can be demonstrated with two resonator modes. We show that this already has implications for weak to moderate coupling.

\section{GENERAL MODEL}

Consider a qubit consisting of an $L C$ oscillator in parallel with a symmetric potential $U\left(\phi_{\mathrm{q}}\right)$ that is coupled to a multimode resonator [cf. Fig. 1(a)]. We denote the qubit Hamiltonian by $H_{\mathrm{q}}$ and the resonator Hamiltonian by $H_{\mathrm{r}}$. They are coupled via the interaction $V$ such that the total Hamiltonian is given by $H=H_{\mathrm{q}}+H_{\mathrm{r}}+V$. Using the unitary freedom of the Hamiltonian formalism, we introduce a gauge parameter $\eta \in[0,1]$ (see Appendix A) that linearly interpolates between a qubit-resonator interaction mediated by the flux variables $\phi_{k}$ (for $\eta=0$ ) and the charge variables $Q_{k}$ (for $\eta=1$ ). We will refer to these extremal cases as the flux and the charge gauge, respectively. For a general gauge, the interaction reads

$$
\begin{aligned}
V(\eta)= & -\sum_{k=1}^{N}\left[\frac{(1-\eta) \phi_{\mathrm{q}} \phi_{k}}{L_{k}}+\frac{\eta Q_{\mathrm{q}} Q_{k}}{C_{\Sigma}}\right] \\
& +(1-\eta)^{2} \sum_{k=1}^{N} \frac{\phi_{\mathrm{q}}^{2}}{2 L_{k}}+\eta^{2} \frac{\left(\sum_{k=1}^{N} Q_{k}\right)^{2}}{2 C_{\Sigma}} ;
\end{aligned}
$$



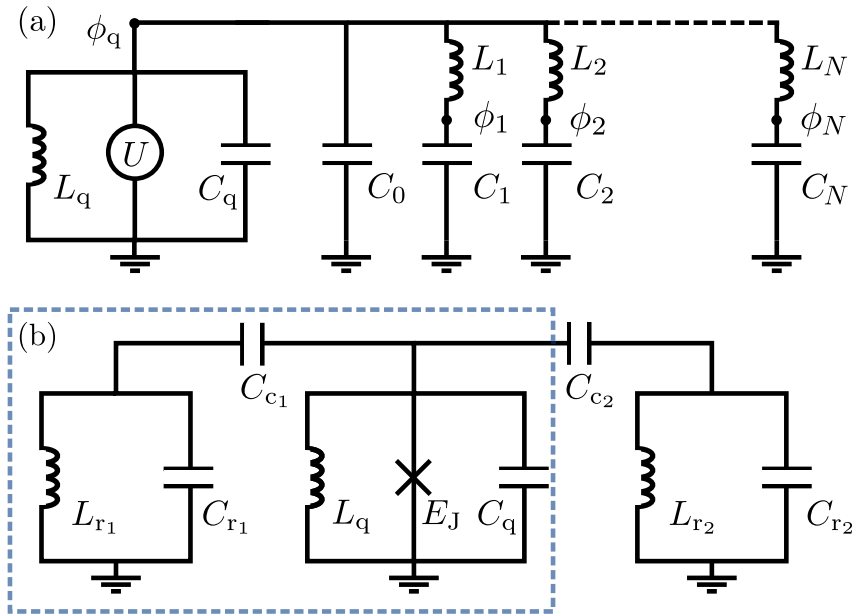

FIG. 1. (a) Circuit diagram of a qubit with potential $U\left(\phi_{\mathrm{q}}\right)$, capacitance $C_{\mathrm{q}}$, and inductance $L_{\mathrm{q}}$ that is coupled to a general reactive environment. In the Foster form, the latter is represented by $N$ resonators with capacitances $C_{k}$ and inductances $L_{k}(k=1, \ldots, N)$. (b) Fluxonium qubit (consisting of a Josephson junction with energy $E_{\mathrm{J}}$ in parallel to the capacitance $C_{\mathrm{q}}$ and a large inductance $L_{\mathrm{q}}$ ), capacitively coupled to two resonators with inductances $L_{r_{k}}$ and capacitances $C_{r_{k}}(k=1,2)$. In the text, the example of a single resonator (dashed box) is treated separately.

here $C_{\Sigma}=C_{\mathrm{q}}+C_{0}$ denotes the total capacitance of the qubit to ground. The first term of Eq. (1) is the analog of the paramagnetic coupling. The second is a diamagnetic term that renormalizes the qubit and the resonator frequencies and ensures the gauge invariance of the full Hamiltonian [24]. In deriving Eq. (1) we have defined the qubit variable to be the one associated with the anharmonic potential $U\left(\phi_{\mathrm{q}}\right)$. The derivation of the full Hamiltonian is detailed in Appendix B.

For most quantum information applications, we are interested in projecting $H_{\mathrm{q}}$ onto a subspace $S=\{|0\rangle,|1\rangle\}$ spanned by the two lowest eigenstates. To obtain an effective Hamiltonian, we apply the SW method resulting in $H_{\text {eff }}=\sum_{j=0}^{K} H_{j}$ to $K$ th order. In Appendix $\mathrm{C}$ we show that the first-order result $H_{0}+H_{1}$ corresponds to the projection of $H$ onto $S$. It is equivalent to the generalized $\mathrm{QRM}^{1}$

$$
\begin{aligned}
H_{\mathrm{QRM}}(\eta)= & -\frac{\hbar \omega_{10}^{\mathrm{q}}}{2} \sigma^{z}+\sum_{k=1}^{N} \hbar \omega_{k} a_{k}^{\dagger} a_{k} \\
& +\hbar \sum_{k=1}^{N}\left[(1-\eta) g_{k}^{\phi} \sigma^{x}\left(a_{k}+a_{k}^{\dagger}\right)+\eta g_{k}^{Q} \sigma^{y}\left(a_{k}-a_{k}^{\dagger}\right)\right],
\end{aligned}
$$

where $\hbar \omega_{n m}^{\mathrm{q}}$ is the energy difference between the $n$th and the $m$ th eigenstate of $H_{\mathrm{q}}$ and $\sigma^{j}(j=x, y, z)$ denote the Pauli operators. In Eq. (2) we have rewritten the variables of the $k$ th resonator mode with frequency $\omega_{k}$ in terms of bosonic creation operators $a_{k}^{\dagger}$ and annihilation operators $a_{k}$. The coupling between the qubit and the $k$ th resonator mode is given by

\footnotetext{
${ }^{1}$ Here the parity symmetry $U\left(-\phi_{\mathrm{q}}\right)=U\left(\phi_{\mathrm{q}}\right)$ is important for giving the selection rules $\left\langle j\left|Q_{\mathrm{q}}\right| j\right\rangle=\left\langle j\left|\phi_{\mathrm{q}}\right| j\right\rangle=0$.
}

$g_{k}^{\phi}=\left\langle 1\left|\phi_{\mathrm{q}}\right| 0\right\rangle \sqrt{Z_{k} / 2 \hbar L_{k}^{2}}$ and $g_{k}^{Q}=\left\langle 1\left|Q_{\mathrm{q}}\right| 0\right\rangle / \sqrt{2 \hbar Z_{k} C_{\Sigma}^{2}}$, where $Z_{k}$ is the characteristic impedance of the $k$ th mode. In deriving Eq. (2) we neglected the diamagnetic shift due to the second term present in Eq. (1) for simplicity. For weak coupling, the diamagnetic shift is irrelevant. In general, it can be accounted for using symplectic diagonalization [25,26].

Restricting the perturbative series of $H_{\text {eff }}$ to any finite order necessarily results in a gauge-dependent model. The source of the gauge dependence of the QRM is that the coupling between the subspace $S$ and its orthogonal complement $S^{\perp}$ is not properly taken into account in the projection. Increasing the order $K$ weakens the gauge dependence [27,28] at the expense of introducing a dressed basis that results in a model that strays quite far from the natural interpretation of the QRM. In this respect, the lowest-order approximation provided by the QRM is an appealing model as it yields a low-energy description without rotating the basis. In the simple effective model (2), choosing a gauge such that the QRM accurately captures the physics of the full Hamiltonian is crucial. We are thus concerned with the task of finding an optimal gauge parameter $\eta_{*}$ such that the differences between the QRM and the full Hamiltonian are minimized.

\section{OPTIMAL GAUGE}

To address this issue, we note that the validity of the QRM is directly proportional to the coupling strength between $S$ and $S^{\perp}$. The higher-order SW terms $H_{j}(j>1)$ can therefore be used as an estimator for the difference between the full model and its effective description as a QRM. Based on this observation, we derive an analytic criterion for the optimal gauge.

In particular, we focus on the second-order term $\mathrm{H}_{2}$, which will provide the largest corrections to $H_{\mathrm{QRM}}$ for weak to moderate coupling; $H_{2}$ is proportional to matrix elements $V_{n m}=\langle n|V| m\rangle$ of the interaction, where $|n\rangle \in S$ and $|m\rangle \in S^{\perp}$. Motivated by Eq. (1), we define the paramagnetic flux coupling operator $G_{k}^{\phi}=\phi_{\mathrm{q}} \phi_{k}^{\mathrm{zp}} / \hbar L_{k}$ and the charge coupling operator $G_{k}^{Q}=Q_{\mathrm{q}} Q_{k}^{\mathrm{zp}} / \hbar C_{\Sigma} .{ }^{2}$ Here we have approximated the resonator matrix elements by their zeropoint fluctuations $\phi_{k}^{\mathrm{zp}} \simeq \sqrt{\hbar Z_{k}}$ and $Q_{k}^{\mathrm{zp}} \simeq \sqrt{\hbar / Z_{k}}$, respectively. In order to estimate the relevance of the flux versus the charge coupling (for the transition $m \mapsto n$ ), we introduce the ratio $f_{n m}=\left[\sum_{k}\left(G_{k}^{\phi}\right)_{n m}\right] /\left[\sum_{k}\left(G_{k}^{Q}\right)_{n m}\right]$. Using the fact that $\left(Q_{\mathrm{q}}\right)_{n m}=i \omega_{n m}^{\mathrm{q}} C_{\Sigma}\left(\phi_{\mathrm{q}}\right)_{n m}$, it can be compactly rewritten as

$$
\left|f_{n m}\right|=\frac{\sum_{k} p_{k} \omega_{k}}{\omega_{n m}^{\mathrm{q}}}=\frac{\bar{\omega}}{\omega_{n m}^{\mathrm{q}}},
$$

where $\bar{\omega}$ is the average of the resonator frequencies $\omega_{k}$ with the weights $p_{k}=Z_{k}^{-1 / 2} / \sum_{l} Z_{l}^{-1 / 2}$.

The interpretation of Eq. (3) is as follows: If $\left|f_{n m}\right| \ll 1$, the coupling between $S$ and $S^{\perp}$ in the flux gauge is much smaller

\footnotetext{
${ }^{2}$ Note that the couplings $G^{\phi}$ and $G^{Q}$ are generalizations of the couplings introduced in Eq. (2) such that $\left\langle 0\left|G^{\phi}\right| 1\right\rangle=-g^{\phi}$ and $\left\langle 0\left|G^{Q}\right| 1\right\rangle=-i g^{Q}$.
} 
than the coupling in the charge gauge. The QRM with $\eta \approx 0$ is therefore a good approximation of the full model, making the flux gauge the preferred choice. However, if $\left|f_{n m}\right| \gg 1$, the coupling of the qubit subspace to higher levels is small in the charge gauge, which thus is the optimal gauge. In the intermediate regime, where $\left|f_{n m}\right| \simeq 1$, both flux and charge variables contribute similarly to the coupling between $S$ and $S^{\perp}$. Consequently, we expect the optimal gauge to be neither the pure charge nor the flux gauge but a mixed gauge with $\eta \neq 0,1$.

For weak qubit-resonator interactions, the dominant contribution to $\mathrm{H}_{2}$ will be due to the coupling of the first and second excited level of the qubit. The character of the coupling of the optimal gauge is therefore mostly determined by the ratio of the frequency difference between the first and second excited qubit level to an effective frequency of the resonator. We conclude that $f_{21}$ of Eq. (3) provides a simple estimation of the optimal coupling. We illustrate these findings with two specific examples in the following.

\section{A. Single resonator}

First, we consider a qubit coupled to a single resonator $(N=1)$. Note that in this case the average frequency $\bar{\omega}$ in Eq. (3) is equal to $\omega_{1}$. For the interaction between the qubit and the resonator mode to be appreciable, we assume that $\omega_{1} \simeq \omega_{10}^{\mathrm{q}}$. Consequently, Eq. (3) yields $\left|f_{21}\right| \simeq \omega_{10}^{\mathrm{q}} / \omega_{21}^{\mathrm{q}}$ and the optimal gauge is solely determined by the properties of the qubit. To reach strong coupling, the qubit has to be anharmonic with $\omega_{10}^{\mathrm{q}} \ll \omega_{21}^{\mathrm{q}}$ [20]. This implies $\left|f_{21}\right| \ll 1$, so we find that the flux gauge is always the optimal gauge for this case.

To demonstrate this result, we numerically study the fluxonium qubit with $E_{\mathrm{L}}=\left(\phi_{0} / 2 \pi\right)^{2} / L_{\mathrm{q}} \lesssim E_{\mathrm{J}}$ and $U\left(\phi_{\mathrm{q}}\right)=$ $-E_{\mathrm{J}} \cos \left[2 \pi\left(\phi_{\mathrm{q}}-\phi_{\mathrm{ext}}\right) / \phi_{0}\right]$ [29]; here $E_{\mathrm{J}}$ is the Josephson energy, $\phi_{0}=h / 2 e$ is the superconducting flux quantum, and $\phi_{\text {ext }}$ is an external magnetic flux threading the superconducting loop. We set the external flux to the degeneracy point $\phi_{\text {ext }}=$ $\frac{1}{2} \phi_{0}$, which results in a symmetric potential. The qubit parameters are chosen such that the qubit is strongly anharmonic with $\omega_{21}^{\mathrm{q}} / \omega_{10}^{\mathrm{q}} \approx 25$ (see Fig. 2 for details). The fluxonium qubit is coupled to a parallel combination of a capacitor $C_{\mathrm{r}_{1}}$ and inductance $L_{\mathrm{r}_{1}}$ which together form a resonator with a frequency $\omega_{1}=\omega_{10}^{\mathrm{q}}$ [cf. Fig. 1(b) (dashed box)]. As is shown in Appendix D, the setup can be mapped to the canonical Foster circuit with $N=1$ depicted in Fig. 1(a).

Figure 2(a) shows the spectrum of the full Hamiltonian (solid) compared to the spectrum of $H_{\mathrm{QRM}}$ (dotted) as a function of $\eta$. The spectra agree well in the flux gauge $(\eta=$ 0 ). For increasing values of $\eta$, that is, for more chargelike gauges, the spectral agreement between truncated and full model decreases. The disagreement is more pronounced in levels with higher energy as they are closer to the energy of the second excited level of the qubit. We observe that $f_{21}$ of Eq. (3) is suitable for estimating the overall tendency for being chargelike or fluxlike. A more quantitative estimate of the optimal coupling $\eta_{*}$ can be obtained by calculating the norm of $\mathrm{H}_{2}$. Based on the discussion surrounding Eq. (3), we expect that $\eta_{*}$ is approximately the $\eta$ for which the norm
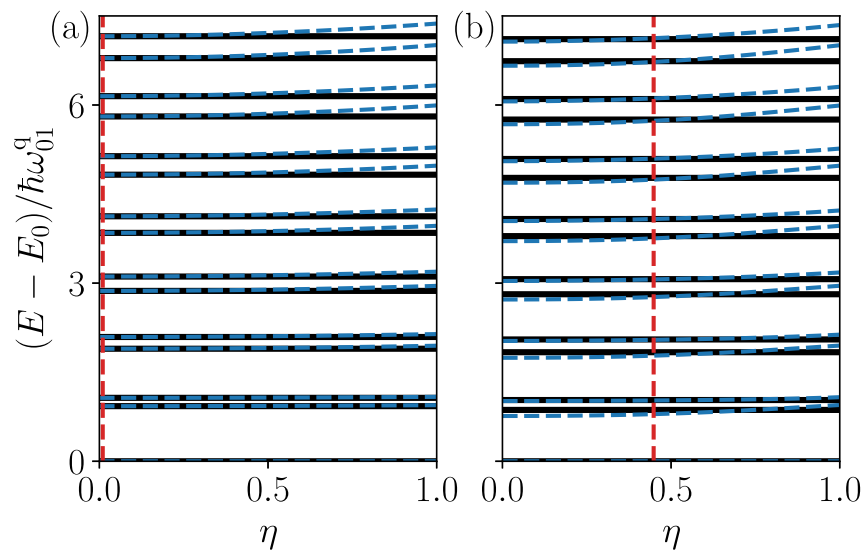

FIG. 2. Spectrum of the full Hamiltonian $H$ (solid lines) and the Rabi model Hamiltonian $H_{\mathrm{QRM}}$ (dashed lines) for a fluxonium qubit coupled to (a) a single resonator and (b) two resonators. The qubit parameters are $\left(E_{\mathrm{J}}, E_{C}, E_{L}\right)=\hbar(12.5,3.75,0.5) \mathrm{GHz}$, where $E_{C}=e^{2} / 2 C_{\Sigma}$ and $E_{\mathrm{L}}=\left(\phi_{0} / 2 \pi\right)^{2} / L_{\mathrm{q}}$. The resulting qubit frequencies are $\omega_{10}^{\mathrm{q}}=0.5 \mathrm{GHz}$ and $\omega_{21}^{\mathrm{q}} \approx 13 \mathrm{GHz}$. Furthermore, $\omega_{1}=\omega_{10}^{q}$ and $g_{1}^{\phi} / \omega_{1}=0.07$. In (b) the parameters of the second resonator are $C_{\mathrm{r}_{2}}=C_{\mathrm{r}_{1}}, C_{\mathrm{c}_{2}}=C_{\mathrm{c}_{1}}$, and $\omega_{2} \approx \omega_{12}^{\mathrm{q}}$ such that $\bar{\omega}=10.7 \mathrm{GHz}$. The value $\eta_{*}$ that minimizes $\left\|H_{2}\right\|_{*}$ is shown as a vertical dashed line.

$\left\|H_{2}\right\|_{*}$ is minimized. ${ }^{3}$ For the parameters in Fig. 2(a), the minimum of $\left\|H_{2}\right\|_{*}$ is at $\eta=0$, which is shown as a red dashed line and agrees well with the visual impression conveyed by the spectrum. A quantitative analysis can be found in Appendix E.

\section{B. Two resonators}

As a second example, we treat the case where there are two relevant modes $(N=2)$. As before, the first mode is close to resonance with the qubit frequency. The second mode with frequency $\omega_{2}$ can be interpreted as a parasitic mode. Since the average frequency $\bar{\omega}$ in Eq. (3) is a function of all modes coupled to the qubit, the optimal gauge is now also dependent on the parasitic mode. This is true even for strongly off-resonant modes, as the coupling to higher modes in the flux gauge increases proportionally to $\left(\omega_{2}\right)^{2}$ at fixed impedance [see Eq. (1)]. As a result, for large detuning with $\omega_{2} \gg \omega_{21}^{\mathrm{q}}$, the charge gauge becomes more favorable. In contrast to the resonant single-mode case, the optimal gauge for two resonators is not determined by the properties of the qubit alone but depends on the parameters of the whole circuit.

To show this effect, we perform numerical simulations of the circuit in Fig. 1(b). The fluxonium is capacitively coupled to two parallel $L C$ oscillators via the capacitances $C_{\mathrm{c}_{1}}$ and $C_{\mathrm{c}_{2}}$. This circuit can be mapped to the canonical Foster circuit depicted in Fig. 1(a) (Appendix D). Figure 2(b) shows the spectrum of the full Hamiltonian (black solid line) and the QRM (blue dashed line) as a function of $\eta$. The

\footnotetext{
${ }^{3}$ In Figs. 2 and 3 we have used the trace norm $\|\mathcal{H}\|_{*}=\sum_{k}\left|\lambda_{k}\right|$ for a Hermitian operator $\mathcal{H}$ with eigenvalues $\lambda_{k}$. Since we are only interested in the value of $\eta$ that minimizes the norm of $\mathrm{H}_{2}$, other norms can be used as well.
} 
parameters of the qubit and the first resonator are the same as in Fig. 2(a). The frequency of the second resonator, however, is significantly larger such that $\bar{\omega} \approx \omega_{12}^{\mathrm{q}}$. In contrast to the single-resonator case, the spectral lines of $H$ and $H_{\mathrm{QRM}}$ do not cross at $\eta \approx 0$ but rather around $\eta \approx 0.5$, suggesting that the optimal gauge does not coincide with the usual ad hoc choices of the flux or charge gauge. This is in agreement with the prediction based on the minimization of $\left\|H_{2}\right\|_{*}$, which yields $\eta_{*}=0.45$ (shown as a dashed vertical line).

The deviation between $H$ and $H_{\mathrm{QRM}}$ is state dependent for finite qubit anharmonicities, a fact that we have neglected so far. As a result, the intersection of the spectral lines of $H$ and $H_{\mathrm{QRM}}$ in Fig. 2(b) is shifted towards smaller values of $\eta$ for increasing energy of the levels. In the intermediate regime where $\omega_{\mathrm{q}}^{21} \simeq \bar{\omega}\left(f_{21} \simeq 1\right)$, the optimal gauge is thus always a compromise which minimizes the differences of $H$ and $H_{\mathrm{QRM}}$ in the relevant spectral range.

To demonstrate the dependence of the optimal gauge on $\bar{\omega}$, we keep the frequency $\omega_{1}$ of the first mode in resonance with the qubit while varying $\bar{\omega}$. To simulate an experimentally feasible scenario, we choose the inductance $L_{r_{2}}$ of the second resonator as the parameter that we vary [30]. Decreasing $L_{\mathrm{r}_{2}}$ while keeping all other parameters constant increases the frequency $\omega_{2}$ of the parasitic mode while simultaneously decreasing its impedance. Since $\bar{\omega}$ decreases with the square root of $Z_{2}$ but increases linearly with $\omega_{2}$, the average frequency
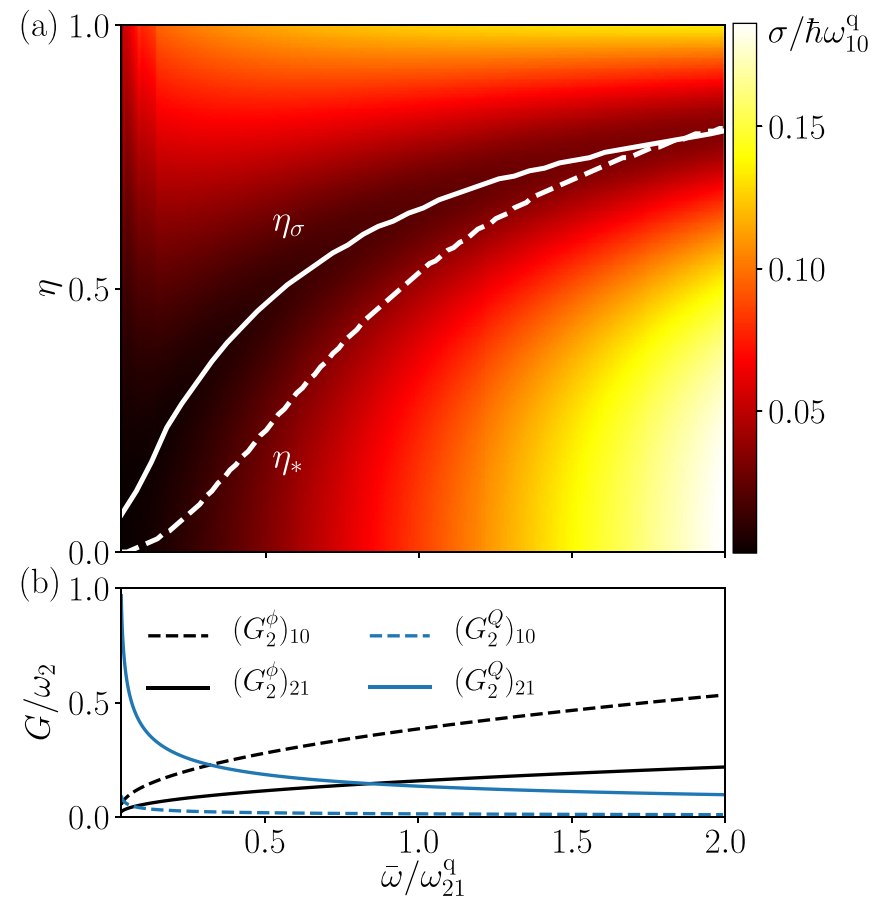

FIG. 3. (a) Deviation $\sigma$ of the energy eigenvalues of the full model from the energy eigenvalues of the QRM as a function of $\eta$ and $\bar{\omega}$. The qubit parameters and the parameters of the first resonator are the same as in Fig. 2. The coupling capacitances to both resonators are equal $C_{\mathrm{c}_{1}}=C_{\mathrm{c}_{2}}=C_{\mathrm{c}}$. The average frequency $\bar{\omega}$ is varied by changing the inductance of the second resonator $L_{\mathrm{r}_{2}}$. The value $\eta_{\sigma}$ for which $\sigma$ is minimized is shown as a solid line. The value of $\eta_{*}$ for which $\left\|H_{2}\right\|_{*}$ is minimized is shown as a dashed line. (b) Coupling strength between qubit levels $n$ and $m$ (see the legend) as a function of $\bar{\omega}$. $\bar{\omega}$ grows with decreasing $L_{r_{2}}$. To quantify the agreement between the full Hamiltonian and the QRM, we use the standard deviation $\sigma=\left[\sum_{i=0}^{M}\left(E_{i}-e_{i}\right)^{2} / M\right]^{1 / 2}$ between the energies $E_{i}$ of the full Hamiltonian and the energies $e_{i}$ of the QRM (measured from the respective ground-state energy). We denote the value of $\eta$ for which $\sigma$ is minimized by $\eta_{\sigma}$.

Figure 3(a) shows $\sigma$ as a function of $\bar{\omega}$ and $\eta$ for $M=10$. We see that $\eta_{\sigma} \approx 0$ (flux gauge) for $\bar{\omega} \ll \omega_{10}^{\mathrm{q}}$. Increasing the average frequency $\bar{\omega}$, the optimal gauge moves towards the charge gauge. Furthermore, we note that although the minimal value of $\sigma$ increases with increasing $\bar{\omega}$, the overall deviation between the full model and the QRM at $\eta_{\sigma}$ is only a few percent. The value $\eta_{*}$ which minimizes $\left\|H_{2}\right\|_{*}$ is shown as a dashed line. It can be observed that $\eta_{*}$ behaves similarly to $\eta_{\sigma}$.

To support our discussion surrounding Eq. (3), we analyze the coupling between $S$ and $S^{\perp}$. Figure 3(b) shows $\left(G_{2}^{\phi}\right)_{n m}$ (black lines) and $\left(G_{2}^{Q}\right)_{n m}$ (blue lines) for the parameters of Fig. 3(a). In general, the charge coupling $G^{Q}$ decreases while the flux coupling $G^{\phi}$ increases with increasing $\bar{\omega}$. For small values of $\bar{\omega}$, the dominant quantity is $\left(G_{2}^{Q}\right)_{21}$. This results in a large coupling between $S$ and $S^{\perp}$ in the charge gauge, making the flux gauge the preferred choice. As $\bar{\omega}$ increases, the coupling to the higher qubit levels in the charge variables decreases and eventually becomes comparable to the coupling in the flux variables, making the choice of the optimal gauge less trivial.

\section{CONCLUSION}

We have analyzed the gauge dependence of the effective description of an anharmonic system coupled to a multimode resonator. Using a SW transformation, we have derived a simple analytic criterion that predicts the optimal gauge where the physics of the nontruncated Hamiltonian is accurately captured by the QRM. We have demonstrated that the optimal gauge for a qubit resonantly coupled to a single resonator is completely determined by the qubit parameters and is in the fluxlike regime for strongly anharmonic qubits. We have seen that coupling a qubit to more than one mode can result in an optimal gauge that is neither the charge nor the flux gauge but a nontrivial combination of the two. This is especially relevant given the increasing interest in the strong qubit-resonator coupling, which raises the need for multimode descriptions. An investigation into the ultrastrong-coupling regime thus constitutes a natural extension of this work in the future.

\section{ACKNOWLEDGMENT}

This work was funded by the Deutsche Forschungsgemeinschaft (DFG, German Research Foundation) under Germany's Excellence Strategy - Cluster of Excellence Matter and Light for Quantum Computing (ML4Q) EXC 2004/1 390534769 .

\section{APPENDIX A: GAUGE TRANSFORMATION}

In this section, we introduce the gauge transformation discussed in the main text on a Lagrangian level. The Lagrangian $\mathcal{L}(\boldsymbol{\phi}, \dot{\boldsymbol{\phi}})$ of a qubit in a potential $U$ coupled to a 
linear (multimode) resonator is a function of the fluxes $\phi=$ $\left(\phi_{\mathrm{q}}, \phi_{1}, \ldots, \phi_{N}\right)^{T}$ and the voltages proportional to $\dot{\boldsymbol{\phi}}$. Here the overdot denotes the time derivative. Using the capacitance matrix $C$ and the inverse of the inductance matrix $M=L^{-1}$, it can be written as

$$
\mathcal{L}(\boldsymbol{\phi}, \dot{\boldsymbol{\phi}})=\frac{1}{2} \dot{\boldsymbol{\phi}}^{T} C \dot{\boldsymbol{\phi}}-\frac{1}{2} \boldsymbol{\phi}^{T} M \boldsymbol{\phi}-U\left(\phi_{\mathrm{q}}\right) .
$$

In Eq. (A1) the flux variable $\phi_{\mathrm{q}}$ is distinguished from the rest by the presence of the potential $U\left(\phi_{\mathrm{q}}\right)$. This allows for a natural assignment of the flux variables to the qubit and the resonator, respectively. We thus consider coordinate transformations $\phi=T \phi^{\prime}$ that preserve this structure and leave the variable $\phi_{\mathrm{q}}$ invariant. In its most general form, such a transformation is given by

$$
T=\left(\begin{array}{ll}
1 & \mathbf{0} \\
\mathbf{t} & R
\end{array}\right),
$$

where $R$ is an invertible matrix and $\mathbf{t}$ is an $N$-dimensional vector.

We now show that the qubit cannot be decoupled from the resonator while simultaneously retaining the structure of Eq. (A1). To demonstrate this, we write $C$ and $M$ in the same block form as Eq. (A2),

$$
C=\left(\begin{array}{ll}
\kappa & \mathbf{c}^{T} \\
\mathbf{c} & C_{\mathrm{r}}
\end{array}\right), \quad M=\left(\begin{array}{cc}
\mu & \mathbf{m}^{T} \\
\mathbf{m} & M_{\mathrm{r}}
\end{array}\right) .
$$

Here $\kappa$ is the qubit capacitance and $\mu$ is the qubit inductance. Moreover, $C_{\mathrm{r}}$ and $M_{\mathrm{r}}$ are the capacitance and inverse inductance matrices of the resonator. The vectors $\mathbf{c}$ and $\mathbf{m}$ couple the flux and voltage variables of the qubit and the resonator. Under the transformation (A2), $C$ and $M$ transform as $C^{\prime}=T^{T} C T$ and $M^{\prime}=T^{T} M T$, which yields the transformed off-diagonal blocks $\mathbf{c}^{\prime}=R \mathbf{c}+R C_{\mathrm{r}} \mathbf{t}$ and $\mathbf{m}^{\prime}=R \mathbf{m}+R M_{\mathrm{r}} \mathbf{t}$. Therefore, in order for $\mathbf{c}^{\prime}$ and $\mathbf{m}^{\prime}$ to vanish at the same time, the following equations have to be satisfied:

$$
\begin{aligned}
& \mathbf{m}-M_{\mathrm{r}} C_{\mathrm{r}}^{-1} \mathbf{c}=0, \\
& \mathbf{c}-C_{\mathrm{r}} M_{\mathrm{r}}^{-1} \mathbf{m}=0 .
\end{aligned}
$$

These equations can only be satisfied simultaneously if the qubit and the resonator are physically decoupled, i.e., if $\mathbf{c}=$ $\mathbf{m}=0$. The transformation (A2) therefore cannot be used to completely decouple the qubit from the resonator. Nevertheless, we can choose coordinates such that the qubit is coupled to the resonator only inductively or capacitively. To this end, we fix $\mathbf{t}$ and introduce a gauge parameter $\eta$ that linearly interpolates between these two extremal cases

$$
\mathbf{t}=-\left[(1-\eta) C_{\mathrm{r}}^{-1} \mathbf{c}+\eta M_{\mathrm{r}}^{-1} \mathbf{m}\right] .
$$

It can be easily verified that $\eta=0$ results in a block-diagonal capacitance matrix $C^{\prime}$. The coupling is then completely inductive and we call the corresponding gauge the flux gauge. On the other hand, $\eta=1$ block diagonalizes $M^{\prime}$, which results in a purely capacitive coupling. We call the corresponding gauge the charge gauge.

\section{APPENDIX B: FULL HAMILTONIAN}

Figure 1(a) in the main text shows a qubit in a potential $U$ coupled to a canonical Foster circuit consisting of a series of
$N L C$ oscillators. With the choice of ground node depicted in this figure, the Lagrangian is given in the flux gauge

$$
\begin{aligned}
\mathcal{L}(\boldsymbol{\phi}, \dot{\boldsymbol{\phi}})= & \frac{C_{\Sigma} \dot{\phi}_{\mathrm{q}}^{2}}{2}-\frac{\phi_{\mathrm{q}}^{2}}{2 L_{\mathrm{q}}}-U\left(\phi_{\mathrm{q}}\right) \\
& +\sum_{k=1}^{N}\left[\frac{C_{k} \dot{\phi}_{k}^{2}}{2}-\frac{\left(\phi_{k}-\phi_{\mathrm{q}}\right)^{2}}{2 L_{k}}\right] .
\end{aligned}
$$

Here $C_{\Sigma}=C_{\mathrm{q}}+C_{0}$ is the total capacitance of the qubit to ground. We introduce a gauge parameter $\eta$ by performing the variable transformation (A2) discussed in Appendix A. We use the specific $\mathbf{t}$ from Eq. (A6). For the Lagrangian in Eq. (B1), the coupling vectors read $\mathbf{c}=\mathbf{0}$ and $\mathbf{m}=\left(-L_{1}^{-1},-L_{2}^{-1}, \ldots,-L_{N}^{-1}\right)^{T}$. The capacitance and inductance matrices of the resonator are diagonal. They are given by $C_{\mathrm{r}}=\operatorname{diag}\left(C_{1}, C_{2}, \ldots, C_{N}\right)$ and $M_{\mathrm{r}}=\operatorname{diag}\left(L_{1}^{-1}, L_{2}^{-1}, \ldots, L_{N}^{-1}\right)$. Performing the transformation yields

$$
\begin{aligned}
\mathcal{L}^{\prime}\left(\boldsymbol{\phi}^{\prime}, \dot{\boldsymbol{\phi}}^{\prime}\right)= & \frac{C_{\Sigma} \dot{\phi}_{\mathrm{q}}^{\prime 2}}{2}-\frac{\phi_{\mathrm{q}}^{\prime 2}}{2 L_{\mathrm{q}}}-U\left(\phi_{\mathrm{q}}^{\prime}\right) \\
& +\sum_{k=1}^{N}\left[\frac{C_{k}\left(\dot{\phi}_{k}^{\prime}+\eta \dot{\phi}_{\mathrm{q}}^{\prime}\right)^{2}}{2}-\frac{\left[\phi_{k}^{\prime}-(1-\eta) \phi_{\mathrm{q}}^{\prime}\right]^{2}}{2 L_{k}}\right],
\end{aligned}
$$

where $\boldsymbol{\phi}=T \boldsymbol{\phi}^{\prime}$. We define the conjugate momenta $Q_{i}^{\prime}=\frac{\partial \mathcal{L}^{\prime}}{\partial \phi_{i}^{\prime}}$ of the flux variables $\boldsymbol{\phi}^{\prime}$ and perform a Legendre transformation which yields the Hamiltonian $H=\sum_{i} Q_{i}^{\prime} \dot{\phi}_{i}^{\prime}-\mathcal{L}^{\prime}$. To obtain a quantum mechanical description, we promote the canonical variables to operators $\phi_{i}^{\prime} \rightarrow \hat{\phi}_{i}$ and $Q_{i}^{\prime} \rightarrow \hat{Q}_{i}$ and impose the canonical commutation relation $\left[\hat{\phi}_{i} \hat{Q}_{j}\right]=i \hbar \delta_{i j}$, where $\delta_{i j}$ is the Kronecker delta. The total Hamiltonian $H(\eta)=H_{\mathrm{q}}+$ $H_{\mathrm{r}}+V(\eta)$ can then be split into a qubit Hamiltonian $H_{\mathrm{q}}$, a resonator Hamiltonian $H_{\mathrm{r}}$, and the interaction $V$ with

$$
\begin{aligned}
H_{\mathrm{q}}= & \frac{\hat{Q}_{\mathrm{q}}^{2}}{2 C_{\Sigma}}+\frac{\hat{\phi}_{\mathrm{q}}^{2}}{2 L_{\mathrm{q}}}+U\left(\hat{\phi}_{\mathrm{q}}\right), \quad H_{\mathrm{r}}=\sum_{k=1}^{N} \frac{\hat{Q}_{k}^{2}}{2 C_{k}}+\frac{\hat{\phi}_{k}^{2}}{2 L_{k}}, \\
V(\eta)= & -\sum_{k=1}^{N}\left[\frac{(1-\eta) \hat{\phi}_{\mathrm{q}} \hat{\phi}_{k}}{L_{k}}+\frac{\eta \hat{Q}_{\mathrm{q}} \hat{Q}_{k}}{C_{\Sigma}}\right] \\
& +(1-\eta)^{2} \sum_{k=1}^{N} \frac{\hat{\phi}_{\mathrm{q}}^{2}}{2 L_{k}}+\eta^{2} \frac{\left(\sum_{k=1}^{N} \hat{Q}_{k}\right)^{2}}{2 C_{\Sigma}} .
\end{aligned}
$$

The interaction $V$ in Eq. (B3b) is given in Eq. (1), where the circumflexes over the operators have been omitted. Note that the Hamiltonian $H(\eta)$ is related to the Hamiltonian $H\left(\eta^{\prime}\right)$ through the unitary transformation $R=\exp \left[-i\left(\eta^{\prime}-\right.\right.$ $\left.\eta) \hat{\phi}_{\mathrm{q}} \sum_{k} \hat{Q}_{k} / \hbar\right]$ such that $R^{\dagger} H(\eta) R=H\left(\eta^{\prime}\right)$. The difference of $H(\eta)-H(0)$ corresponds to a pseudopertubation of Ref. [28].

\section{APPENDIX C: SCHRIEFFER-WOLFF TRANSFORMATION}

In this Appendix we perform a SW transformation to derive the QRM (2). Similar to the main text, we define the low-energy subspace of the qubit $S=\{|0\rangle,|1\rangle\}$ and its 
orthogonal complement $S^{\perp}$. Furthermore, we define the projector $P=|0\rangle\langle 0|+| 1\rangle\langle 1|$ onto $S$. The projector onto $S^{\perp}$ is then given by $Q=1-P$. The coupling between the subspaces $S$ and $S^{\perp}$ is provided by $P V Q$. Performing a SW transformation to block diagonalize $H$ with respect to $S$ and $S^{\perp}$ results in an effective Hamiltonian $H_{\text {eff }}=\sum_{j=0}^{K} H_{j}$ [31]. The zeroth order is given by the projection of the uncoupled Hamiltonian onto $S$,

$$
H_{0}=P H_{\mathrm{q}} P+H_{\mathrm{r}}=-\hbar \frac{\omega_{10}^{\mathrm{q}}}{2} \sigma^{z}+\hbar \sum_{k=1}^{N} \omega_{k} a_{k}^{\dagger} a_{k} .
$$

Here $\hbar \omega_{10}^{\mathrm{q}}$ is the energy difference between the ground state and the first excited state of the qubit. Furthermore, we have defined the frequencies $\omega_{k}=1 / \sqrt{L_{k} C_{k}}$ and the bosonic raising and lowering operators of the $k$ th mode

$$
\begin{aligned}
\hat{\phi}_{n} & =\sqrt{\frac{\hbar Z_{k}}{2}}\left(a_{k}^{\dagger}+a_{k}\right), \\
\hat{Q}_{k} & =i \sqrt{\frac{\hbar}{2 Z_{k}}}\left(a_{k}^{\dagger}-a_{k}\right),
\end{aligned}
$$

where $Z_{k}=\sqrt{L_{k} / C_{k}}$ is the characteristic impedance of the $k$ th mode. The next order is given by the projection of the interaction $V$ onto $S$,

$$
\begin{aligned}
H_{1}= & P V(\eta) P \\
= & \hbar \sum_{k=1}^{N}\left[(1-\eta) g_{k}^{\phi} \sigma^{x}\left(a_{k}+a_{k}^{\dagger}\right)+\eta g_{k}^{Q} \sigma^{y}\left(a_{k}-a_{k}^{\dagger}\right)\right] \\
& -\frac{(1-\eta) \alpha}{2} \sigma^{z}-\frac{\eta^{2} \hbar}{2 C_{\Sigma}}\left(\sum_{k=1}^{N} \frac{a_{k}^{\dagger}-a_{k}}{\sqrt{Z_{k}}}\right)^{2},
\end{aligned}
$$

where $\quad g_{k}^{\phi}=\left\langle 1\left|\phi_{\mathrm{q}}\right| 0\right\rangle \sqrt{Z_{k} / 2 \hbar L_{k}^{2}} \quad$ and $\quad g_{k}^{Q}=$ $\left\langle 1\left|Q_{\mathrm{q}}\right| 0\right\rangle / \sqrt{2 \hbar Z_{k} C_{\Sigma}^{2}}$. Furthermore, $\quad \alpha=\left(\left\langle 1\left|\phi_{\mathrm{q}}^{2}\right| 1\right\rangle-\right.$ $\left.\left\langle 0\left|\phi_{\mathrm{q}}^{2}\right| 0\right\rangle\right) \sum_{n} 1 / L_{n}$. The last two terms in Eq. (C4) are diamagnetic renormalizations of the qubit and resonator frequencies. If these terms are omitted, the first-order effective Hamiltonian $H_{0}+H_{1}$ is equal to the QRM (2). For weak qubit-resonator coupling this is a reasonable assumption.

\section{APPENDIX D: FOSTER REPRESENTATION}

The circuit shown in Fig. 1(b) can be mapped onto the general Foster form of Fig. 1(a). Assuming a symmetric coupling $C_{\mathrm{c}_{1}}=C_{\mathrm{c}_{2}}=C_{\mathrm{c}}$, the capacitances and inductances are given by the substitutions

$$
C_{k}=\frac{C_{\mathrm{c}}^{2}}{C_{\mathrm{c}}+C_{r_{k}}}, \quad L_{k}=\frac{L_{r_{k}}\left(C_{\mathrm{c}}+C_{r_{k}}\right)^{2}}{C_{\mathrm{c}}^{2}},
$$

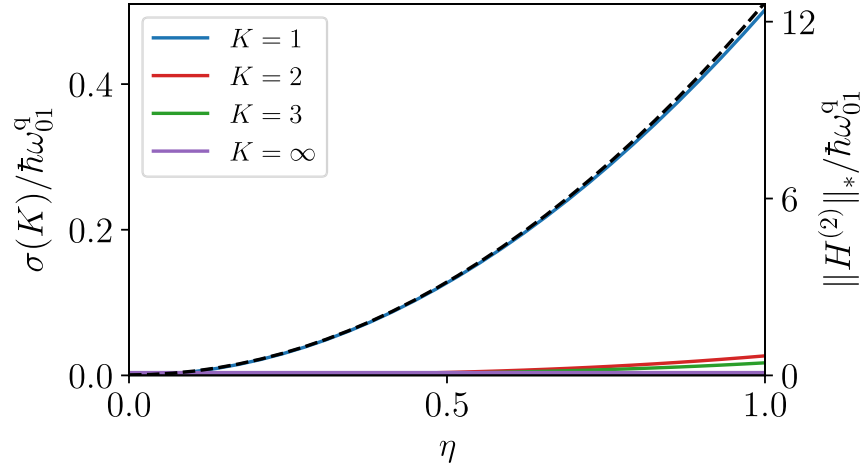

FIG. 4. Standard deviation $\sigma$ of the full spectrum and the effective Hamiltonian $H_{\text {eff }}$ to $K$ th order. The QRM corresponds to $K=1$ (blue). Moreover, $K=2$ (red), $K=3$ (green), and the exact SW transformed Hamiltonian $K=\infty$ (purple) are shown. Additionally, the norm $\left\|H_{2}\right\|_{*}$ of the first perturbative correction to $H_{\mathrm{QRM}}$ is shown (black dashed line). The parameters are the same as in Fig. 2.

with

$$
C_{0}=\frac{C_{\mathrm{c}} C_{r_{1}}}{C_{\mathrm{c}}+C_{r_{1}}}
$$

for the one-mode setup (dashed box) and

$$
C_{0}=C_{\mathrm{c}}\left(\frac{C_{r_{1}}}{C_{\mathrm{c}}+C_{r_{1}}}+\frac{C_{r_{2}}}{C_{\mathrm{c}}+C_{r_{2}}}\right)
$$

for the two-mode setup.

\section{APPENDIX E: DETAILS OF THE SINGLE RESONATOR RESULTS}

In this Appendix we show supporting data for one qubit coupled to one resonator. Figure 4 shows the standard deviation $\sigma(K)=\sqrt{(1 / M) \sum_{i=0}^{M}\left[E_{i}-e_{i}(K)\right]^{2}}$ for the first ten states. Note that here $e_{i}(K)$ denote the eigenvalues of the SW transformed Hamiltonian $H_{\text {eff }}$ to $K$ th order. In Fig. 4 the parameters are the same as in Fig. 2. For all finite values of $K$, the minimum of $\sigma$ is at $\eta \approx 0$, demonstrating that the flux gauge is optimal in this case.

Furthermore, we see that adding higher-order terms to the Rabi Hamiltonian mitigates the effect of the broken gauge invariance. The deviation between the full and the effective model becomes less sensitive to variations in $\eta$ with increasing order in the SW method. The exact SW transformation [27] (purple solid line) results in a gauge invariant two-level description. Additionally, the norm of $\mathrm{H}_{2}$ is shown (black dashed line). We observe a nonlinear increase towards chargelike gauges, as expected from the discussion in Sec. III A. For $K=$ 1 (blue solid line), we see a similar functional dependence on $\eta$ as in $\left\|H_{2}\right\|_{*}$, indicating that many of the corrections to $H_{\mathrm{QRM}}$ are already captured by $\mathrm{H}_{2}$.
[1] A. Blais, R.-S. Huang, A. Wallraff, S. M. Girvin, and R. J. Schoelkopf, Cavity quantum electrodynamics for superconduct- ing electrical circuits: An architecture for quantum computation, Phys. Rev. A 69, 062320 (2004). 
[2] A. Wallraff, D. I. Schuster, A. Blais, L. Frunzio, R.-S. Huang, J. Majer, S. Kumar, S. M. Girvin, and R. J. Schoelkopf, Strong coupling of a single photon to a superconducting qubit using circuit quantum electrodynamics, Nature (London) 431, 162 (2004).

[3] S. Schmidt and J. Koch, Circuit QED lattices: Towards quantum simulation with superconducting circuits, Ann. Phys. (Berlin) 525, 395 (2013).

[4] M. H. Devoret and R. J. Schoelkopf, Superconducting circuits for quantum information: An outlook, Science 339, 1169 (2013).

[5] G. Wendin, Quantum information processing with superconducting circuits: A review, Rep. Prog. Phys. 80, 106001 (2017).

[6] J. R. Schrieffer and P. A. Wolff, Relation between the Anderson and Kondo Hamiltonians, Phys. Rev. 149, 491 (1966).

[7] S. Bravyi, D. P. DiVincenzo, and D. Loss, Schrieffer-Wolff transformation for quantum many-body systems, Ann. Phys. (NY) 326, 2793 (2011).

[8] W. E. Lamb, Fine structure of the hydrogen atom. III, Phys. Rev. 85, 259 (1952).

[9] K.-H. Yang, Gauge transformations and quantum mechanics I. Gauge invariant interpretation of quantum mechanics, Ann. Phys. (NY) 101, 62 (1976).

[10] D. De Bernardis, P. Pilar, T. Jaako, S. De Liberato, and P. Rabl, Breakdown of gauge invariance in ultrastrong-coupling cavity QED, Phys. Rev. A 98, 053819 (2018).

[11] D. De Bernardis, T. Jaako, and P. Rabl, Cavity quantum electrodynamics in the nonperturbative regime, Phys. Rev. A 97, 043820 (2018).

[12] O. Di Stefano, A. Settineri, V. Macrì, L. Garziano, R. Stassi, S. Savasta, and F. Nori, Resolution of gauge ambiguities in ultrastrong-coupling cavity quantum electrodynamics, Nat. Phys. 15, 803 (2019).

[13] S. E. Nigg, H. Paik, B. Vlastakis, G. Kirchmair, S. Shankar, L. Frunzio, M. H. Devoret, R. J. Schoelkopf, and S. M. Girvin, Black-Box Superconducting Circuit Quantization, Phys. Rev. Lett. 108, 240502 (2012).

[14] F. Solgun, D. W. Abraham, and D. P. DiVincenzo, Blackbox quantization of superconducting circuits using exact impedance synthesis, Phys. Rev. B 90, 134504 (2014).

[15] A. Parra-Rodriguez, E. Rico, E. Solano, and I. L. Egusquiza, Quantum networks in divergence-free circuit QED, Quantum Sci. Technol. 3, 024012 (2018).

[16] C. Sánchez Muñoz, F. Nori, and S. De Liberato, Resolution of superluminal signaling in non-perturbative cavity quantum electrodynamics, Nat. Commun. 9, 1924 (2018).
[17] F. Hassler, J. Stubenrauch, and A. Ciani, Equation of motion approach to black-box quantization: Taming the multimode Jaynes-Cummings model, Phys. Rev. B 99, 014515 (2019).

[18] M. F. Gely, A. Parra-Rodriguez, D. Bothner, Y. M. Blanter, S. J. Bosman, E. Solano, and G. A. Steele, Convergence of the multimode quantum Rabi model of circuit quantum electrodynamics, Phys. Rev. B 95, 245115 (2017).

[19] S. J. Bosman, M. F. Gely, V. Singh, A. Bruno, D. Bothner, and G. A. Steele, Multi-mode ultra-strong coupling in circuit quantum electrodynamics, npj Quantum Inf. 3, 46 (2017).

[20] V. E. Manucharyan, A. Baksic, and C. Ciuti, Resilience of the quantum Rabi model in circuit QED, J. Phys. A: Math. Theor 50, 294001 (2017).

[21] A. Frisk Kockum, A. Miranowicz, S. De Liberato, S. Savasta, and F. Nori, Ultrastrong coupling between light and matter, Nat. Rev. Phys. 1, 19 (2019).

[22] A. Stokes and A. Nazir, Gauge ambiguities imply JaynesCummings physics remains valid in ultrastrong coupling QED, Nat. Commun. 10, 499 (2019).

[23] C. CohenTannoudji, J. DupontRoc, and G. Grynberg, Photons and Atoms (Wiley-VCH, Weinheim, 1999).

[24] M. Malekakhlagh and H. E. Türeci, Origin and implications of an $A^{2}$-like contribution in the quantization of circuit-QED systems, Phys. Rev. A 93, 012120 (2016).

[25] M. Idel, S. S. Gaona, and M. M. Wolf, Perturbation bounds for Williamson's symplectic normal form, Linear Algebra App. 525, 45 (2017).

[26] R. Simon, S. Chaturvedi, and V. Srinivasan, Congruences and canonical forms for a positive matrix: Application to the Schweinler-Wigner extremum principle, J. Math. Phys. 40, 3632 (1999).

[27] L. S. Cederbaum, J. Schirmer, and H. D. Meyer, Block diagonalisation of Hermitian matrices, J. Phys. A: Math. Gen. 22 2427 (1989).

[28] Y. Aharonov and C. K. Au, Gauge invariance and pseudoperturbations, Phys. Rev. A 20, 1553 (1979).

[29] V. E. Manucharyan, J. Koch, L. I. Glazman, and M. H. Devoret, Fluxonium: Single cooper-pair circuit free of charge offsets, Science 326, 113 (2009).

[30] M. A. Castellanos-Beltran, K. D. Irwin, G. C. Hilton, L. R. Vale, and K. W. Lehnert, Amplification and squeezing of quantum noise with a tunable Josephson metamaterial, Nat. Phys. 4, 929 (2008).

[31] R. Winkler, Spin-Orbit Coupling Effects in Two-Dimensional Electron and Hole System (Springer, Berlin, 2003), pp. 201-205. 